This is an author-created, un-copyedited version of an article published in Physics in Medicine \& Biology. IOP Publishing Ltd is not responsible for any errors or omissions in this version of the manuscript or any version derived from it. The Version of Record is available online at https://doi.org/10.1088/1361-6560/aaf96c.

\title{
Predicting tumour response to anti-PD-1 immunotherapy with computational modelling
}

Damijan Valentinuzzi ${ }^{1,2}$, Urban Simončič ${ }^{1,2}$, Katja Uršič́3 ${ }^{3}$, Martina Vrankar $^{3}$, Maruša Turk ${ }^{2}$ and Robert Jeraj ${ }^{1,2,4}$

${ }^{1}$ Jožef Stefan Institute, Ljubljana, Slovenia, EU

${ }^{2}$ Faculty of Mathematics and Physics, University of Ljubljana, Ljubljana, Slovenia, EU

${ }^{3}$ Institute of Oncology Ljubljana, Ljubljana, Slovenia, EU

10

${ }^{4}$ Department of Medical Physics, University of Wisconsin, Madison, WI, USA

e-mail: damijan.valentinuzzi@,ijs.si

\begin{abstract}
Cancer immunotherapy is a rapidly developing field, with numerous drugs and therapy combinations waiting to be tested in pre-clinical and clinical settings. However, the costly and time-consuming trial-and-error approach to development of new treatment paradigms creates a research bottleneck, motivating the development of complementary approaches. Computational modelling is a compelling candidate for this task, however, difficulties associated with the validation of such models limits their use in pre-clinical and clinical settings. Here we propose a bottom-up deterministic computational model to simulate tumour response to treatment with anti-programmed-death-1 antibodies (antiPD-1). The model was built with validation in mind, and so contains minimum number of parameters, and only 4 free parameters. Moreover, all model parameters can be measured experimentally. Free parameters were tuned by fitting the model to experimental data from the literature, using B16-F10 murine melanoma implanted into wild type (C57BL/6), as well as into immunodeficient (NSG) mice strains, and treated with anti-PD-1 antibodies. The model's predictive ability was verified on two independent datasets from literature with different but well-known inputs. To identify possible biomarkers of response to anti-PD-1 immunotherapy, sensitivity study of key model parameters was performed. Good agreement between the simulated tumour growth curves and the experimental data was achieved, with mean relative deviations in the range of 13
\end{abstract}


$-20 \%$. Our sensitivity study demonstrated that major histocompatibility complex (MHC) class I expression was the only parameter able to clearly discriminate responders from non-responders to anti-PD-1 therapy. Additionally, the results of sensitivity studies suggest that MHC class I expression might affect the predictive ability of other biomarkers that are currently used in the clinics, such as PD-1 ligand (PD-L1) expression. Interestingly, our model predicts the best response to anti-PD-1 therapy for subjects with moderate PD-L1 values. Such computational models show promise to support, guide and accelerate future immunotherapy research.

Keywords: computational model, mathematical model, tumour modelling, tumour growth, tumour biology, T cells, anti-PD-1, immunotherapy, MHC class I, PD-L1, B16 melanoma

\section{Classification numbers:}

87.17.Aa (Modelling, computer simulation of cell processes)

87.19.xj (Cancer) 


\section{Introduction}

Immunotherapy with immune checkpoint inhibitors (ICI) has become a cornerstone of modern cancer therapy, resulting in favourable outcomes and long-term remissions for a percentage of metastatic patients. Monoclonal antibodies $(\mathrm{mAb})$ targeting receptors on $\mathrm{T}$ cells, tumour cells or antigen-presenting cells, such as cytotoxic T lymphocyte-associated antigen 4 (CTLA-4) and programmed-death-1 (PD-1) and its ligand (PD-L1) have proven to be highly efficient for treatment of melanoma (Ribas et al. 2015), non-small-cell lung cancer (Garon et al. 2015), renal-cell carcinoma (Motzer et al. 2015) and other malignancies, leading to US Food and Drug Administration (FDA) and European Medicines Agency (EMA) approval of ipilimumab (CTLA-4 inhibitor), pembrolizumab, nivolumab (both PD-1 inhibitors), as well as atezolizumab, avelumab and durvalumab (the latter FDA only) (all PD-L1 inhibitors).

However, despite the fact that ICI response rates can be as high as $40 \%$ (e.g. in metastatic

65 melanoma), a majority of patients still fail to respond. Biologic and molecular mechanisms of intrinsic and acquired resistance to ICI are under intense investigation, but the causes of patient non-response still remain largely unknown (Kelderman et al. 2014; Pulluri et al. 2017). There are ongoing efforts to discover pre-treatment biomarkers of response to ICI, as this would allow for the identification of patients that are most likely to respond, and consequently prevent other 70 patients from unnecessary immune-related adverse events (Sharma \& Allison 2015). Furthermore, due to unconventional response patterns (e.g. pseudoprogression) not usually observed in classical cytotoxic therapies, new guidelines for evaluation of efficacy of immunotherapies, such as iRECIST, are being constantly developed (Seymour et al. 2017).

Since it has become clear that most currently available immunotherapies are highly

75 beneficial for just a minority of patients when given as monotherapies, combination strategies are already being tested in various pre-clinical and clinical trials to enhance the efficacy of immunotherapies. For instance, besides combining two or more ICI (e.g. anti-PD-1 and antiCTLA-4), these drugs can also be combined with other ICI (e.g. inhibitors of LAG-3 (CD223), TIM-3, VISTA, BTLA (CD272)), agonist antibodies (targeting OX40 (CD134), 4-1BB (CD137), 
80 ICOS (CD278)), cytokines (IL-2, IL-10, IL-12), different cancer vaccines, oncolytic viruses, or conventional therapies, such as surgery, chemotherapy, radiotherapy, anti-angiogenic therapy, and hormonal therapy (Tsiatas et al. 2016; Sharma \& Allison 2015). The number of all possible combinations greatly exceeds the ability to test all of these combinations in pre-clinical and clinical settings by using a standard trial-and-error approach.

Therefore, development of novel tools such as computational models that are able to predict possible outcomes of different monotherapies and therapy combinations quickly, could tremendously help to (1) identify the most promising candidate therapies to be tested in preclinical and clinical trials, (2) identify the most important biological mechanisms, which could help select new candidates for biomarkers of response to immunotherapy, or (3) provide clues about the dynamics of complex biological processes and mechanisms responsible for tumour development, which might explain the dynamics of unusual tumour response patterns, such as pseudoprogression.

The first computational and mathematical models of different cancer immunotherapies appeared in the 1990s (dePillis et al. 2014; Agur et al. 2016). The earliest model we identified was

95 by Nani and Oğuztöreli, who developed a mathematical model to simulate tumour response to adoptive cell transfer (ACT) immunotherapy (Nani \& Oğuztöreli 1994). In 1998 Kirschner and Panetta simulated ACT and the cytokine interleukin-2 (IL-2) immunotherapies, both as monotherapies as well as a combined treatment (Kirschner \& Panetta 1998). Many different immunotherapy models and modelling approaches followed in the next three decades, however,

100 their use in clinical settings is still limited. Although the understanding of the biological processes involved in immunotherapy has increased considerably up to now, the main obstacles and limitations remain similar. They include the extreme complexity of the immune system and a lack of high-quality, quantitative experimental data to enable the tuning of a computational model. Therefore when developing such models, trade-offs must be made between capturing immune 105 complexity with a risk of losing predictive power because of too many free parameters, and building simple quantifiable models with a risk of losing predictive power due to an oversimplification of physical reality. 
There are several interesting models simulating response to anti-PD-1 therapy. For example, Gong and colleagues developed a multiscale agent-based model able to reproduce 110 spatiotemporal dynamics of $\mathrm{T}$ cells and cancer cells during tumour progression and during PD1/PD-L1 pathway inhibition (Gong et al. 2017). Serre and colleagues developed a top-down pharmacodynamic model simulating synergy between radiotherapy and concurrent PD-1/PD-L1 and CTLA-4 blockade (Serre et al. 2016). Lai and Friedman published two papers: one aimed to simulate the correlation between BRAF/MEK inhibitor and PD-1 inhibitor for the treatment of 115 melanoma (Lai \& Friedman 2017a), and the other aimed to simulate efficacy of the combination of a GM-CSF-secreting cancer vaccine (GVAX) and anti-PD-1 antibodies (Lai \& Friedman 2017b). Recently, Kosinsky and colleagues published a quantitative systems pharmacology model to simulate radiotherapy and anti-PD-(L)1 treatment combinations (Kosinsky et al. 2018). With the ability to describe the studied phenomena, each of these models represents a step forward in an effort to develop novel tools for simulating immunotherapy. However, they all consist of a relatively high number of model parameters, many of which are considered free. For example, the ratio of total number of model parameters to free model parameters in the aforementioned models is as follows: 29/13 (at least) (Gong et al. 2017), 13/13 (Serre et al. 2016), 72/46 (at least) (Lai \& Friedman 2017a), 48/26 (at least) (Lai \& Friedman 2017b), 25/8 (at least) (Kosinsky et al. 2018).

125 This raises doubts as to whether such models are really able to predict outcome in independent experiments, possibly in different situations, or if modelling results resembling experimental data are just a consequence of overfitting.

Our main aim was to build a bottom-up computational model from basic biological principles with a minimum yet reasonable number of parameters, which could also be 130 experimentally quantified. Once the model was formulated, we used one set of data to tune the model, and then evaluated model predictions on two independent datasets with different, but wellknown model inputs. Lastly, we performed sensitivity study of key model parameters with an aim to identify and predict possible biomarkers of response to anti-PD-1 immunotherapy. 


\section{2. Methods}

\subsection{Model Formulation}

The basic idea for constructing a model was adopted from a paper by Kuznetsov and Knott (Kuznetsov \& Knott 2001). They proposed a simple deterministic population model, describing the interplay between tumour cells and cytotoxic killer cells. In our model, the role of cytotoxic

140 killer cells is assigned to tumour infiltrating lymphocytes TILs. Besides the interplay between tumour cells $C$ (Eq. 1) and TILs (Eq. 2), we additionally take into account the dynamics of dead tumour cells $C_{\text {dead }}$. Dead tumour cells are not removed from the tumour instantly and therefore contribute to the overall tumour volume (Eq. 3). Tumour volume $V$ is calculated as described in Eq. 4. The main enhancement of our model (Eq. $1-4)$ as compared to the model of Kuznetsov

145 and colleagues is the inclusion of the most important biological properties and processes involved in anti-PD-1 immunotherapy, such as tumour mutational burden (as reflected by single nucleotide variations (SNV)), tumour ability to present antigens (MHC class I), and tumour ability to suppress immune response (PD-1/PD-L1 pathway - the target of anti-PD-1 immunotherapy). All of these biological properties were found promising for predicting response to anti-PD-1 immunotherapy

150 in various types of cancer (Cristescu et al. 2018; Zaretsky et al. 2016). Additionally, these biological properties are incorporated as measurable parameters in our model, therefore the model is reasonably close to the optimal for our task. All other indispensable parameters were carefully assessed from the available literature. If a literature value could not be obtained, parameters remained free and were used to fit the model. We are aware that more complex models describing 155 additional components of the immune system (dendritic cells, other T cell subpopulations, immune suppressive cells, innate immune system, etc.) would theoretically provide a better description of reality, but at a cost of additional free parameters. This would most likely lead to overfitting. Nevertheless, the effects of other immune system components on tumour growth or regression can be recognized indirectly in our model, namely through the increase or decrease in the values of

160 fitted parameters (in this case interpreted as macroparameters). All model parameters, their values and the corresponding references are summarized in Table 1. 


$$
\begin{aligned}
& \frac{d C}{d t}=k \cdot C \cdot \ln \left(\frac{C_{\text {max }}}{C+C_{\text {dead }}}\right)-a \cdot S N V \cdot M H C I \cdot\left(1-P D L 1 \cdot P D 1 \cdot\left(1-P D 1_{\text {occup }}(D, t)\right)\right) \cdot T I L \cdot C \\
& \frac{d T I L}{d t}= \\
& =b \cdot S N V \cdot\left(C+C_{\text {dead }}\right)^{\frac{2}{3}}-a \cdot S N V \cdot M H C I \cdot P D L 1 \cdot P D 1 \cdot\left(1-P D 1_{\text {occup }}(D, t)\right) \cdot T I L \cdot C-d \cdot T I L
\end{aligned}
$$

$$
\begin{aligned}
& \frac{d C_{\text {dead }}}{d t}=a \cdot S N V \cdot M H C I \cdot\left(1-P D L 1 \cdot P D 1 \cdot\left(1-P D 1_{\text {occup }}(D, t)\right)\right) \cdot T I L \cdot C-I \cdot C_{\text {dead }} \\
& V(t)=V_{c} \cdot\left(C(t)+C_{\text {dead }}(t)\right)+V_{T I L} \cdot \operatorname{TIL}(t)=\frac{4}{3} \pi r_{c}^{3} \cdot\left(C(t)+C_{\text {dead }}(t)\right)+\frac{4}{3} \pi r_{T I L}^{3} \cdot T I L(t)
\end{aligned}
$$

\begin{tabular}{|c|c|c|c|c|}
\hline VARIABLE & DESCRIPTION & & EQUATION & \\
\hline C & Number of live tumour cells & & Eq. 1 & \\
\hline$T I L$ & $\begin{array}{l}\text { Number of tumour infiltrating } \\
\text { lymphocytes }\end{array}$ & & Eq. 2 & \\
\hline$C_{\text {dead }}$ & Number of dead tumour cells & & Eq. 3 & \\
\hline$V$ & Tumour volume & & Eq. 4 & \\
\hline PARAMETER & DESCRIPTION & VALUE & REFERENCE & \\
\hline$k$ & Tumour growth rate & 0.038 day $^{-1}$ & $\begin{array}{l}\text { Tuned to experimental data } \\
\text { (Kleffel et al. 2015) }\end{array}$ & \multirow{4}{*}{ 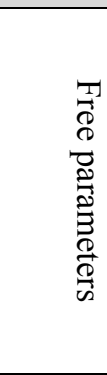 } \\
\hline$C_{\max }$ & $\begin{array}{c}\text { Maximum number of cells in } \\
\text { tumour }\end{array}$ & $1.45 \times 10^{11}$ & $\begin{array}{l}\text { Tuned to experimental data } \\
\text { (Kleffel et al. 2015) }\end{array}$ & \\
\hline$a$ & $\begin{array}{c}\text { Interaction rate between tumour } \\
\text { cells and tumour infiltrating } \\
\text { lymphocytes }\end{array}$ & $\begin{array}{l}5.64 \times 10^{-9} \\
\text { day }^{-1}\end{array}$ & $\begin{array}{l}\text { Tuned to experimental data } \\
\text { (Kleffel et al. 2015) }\end{array}$ & \\
\hline$b$ & $\begin{array}{c}\text { Infiltration rate of T cells into } \\
\text { tumour }\end{array}$ & $\begin{array}{c}7.63 \times 10^{-3} \\
\text { day }^{-1}\end{array}$ & $\begin{array}{c}\text { Tuned to experimental data } \\
\text { (Kleffel et al. 2015) }\end{array}$ & \\
\hline$S N V$ & Single nucleotide variations & 908 & (Castle et al. 2015) & \multirow{9}{*}{ 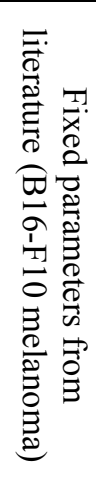 } \\
\hline$M H C I$ & MHC class I expression & $2.3 \%$ & (Yamamura et al. 2015) & \\
\hline PDL1 & PD-L1 expression & $37.5 \%$ & (Kleffel et al. 2015) & \\
\hline$P D 1$ & PD-1 expression & $54 \%$ & (Kodumudi et al. 2016) & \\
\hline PD1occup & $\begin{array}{l}\text { Occupancy of PD- } 1 \text { by anti-PD-1 } \\
\text { antibodies }\end{array}$ & $\begin{array}{l}\text { dose- and } \\
\text { schedule- } \\
\text { dependent }\end{array}$ & (Brahmer et al. 2010) & \\
\hline I & Dead tumour cells removal rate & 0.0935 day $^{-1}$ & (Chvetsov 2013) & \\
\hline$d$ & Natural TIL death rate & 0.406 day $^{-1}$ & (De Boer et al. 2003) & \\
\hline$r_{c}$ & tumour cell radius & $11.4 \mu \mathrm{m}$ & (Rems et al. 2013) & \\
\hline$r_{T T L}$ & TIL radius & $5 \mu \mathrm{m}$ & (Gakamsky et al. 2005) & \\
\hline
\end{tabular}

Table 1: List of model variables and parameters used for tuning the model, with the corresponding references.

Short description of the terms in the model equations:

- Eq. 1, first term: Gompertz function - intrinsic tumour growth in the absence of the immune system.

- Eq. 1, second term: tumour cell loss due to the interaction with the immune system.

- Eq. 2, first term: infiltration of TILs into the tumour. 
- Eq. 2, second term: TIL loss due to the interaction with tumour cells.

- Eq. 2, third term: natural death of TILs.

- Eq. 3, first term: Appearance of dead tumour cells (see Eq. 1)

- Eq. 3, second term: Removal of dead tumour cells from the tumour.

- Eq. 4, first term: Total volume of tumour cells.

- Eq. 4, second term: Total volume of TILs.

The model was constructed on the basis of the following biological rationale and the underlying

180 assumptions:

\section{1) Intrinsic tumour growth follows Gompertz function.}

Uncontrolled sustained proliferation has long been known as one of the hallmarks of cancer (Hanahan \& Weinberg 2000; Hanahan \& Weinberg 2011). Several mathematical functions with different degrees of complexity are commonly used to describe intrinsic tumour growth:

185 exponential, exponential linear, power law, logistic, generalized logistic, von Bertalanffy, Gompertz, etc. In our model, we have chosen Gompertz function, because it was shown that in most situations, it represents the best trade-off between the goodness of fit to the experimental data and the complexity of a modelling function (number of parameters) (Benzekry et al. 2014).

2) Only $T$ cells are involved in anti-tumour response to anti-PD-1 immunotherapy. Anti-PD-1 immunotherapy is typically considered to enhance T cell response (Pardoll 2012; Topalian et al. 2012). Consequently, we assume that only $\mathrm{T}$ cells infiltrating the tumour, i.e. tumour infiltrating lymphocytes (TILs), are involved in anti-tumour response to anti-PD-1 immunotherapy. PD-1 can also be expressed on the surface of other immune effector cells, such as natural killer (NK) cells, but as a first approximation we neglect the antitumour activity of NK cells in anti-PD-1 immunotherapy (Norris et al. 2012). If the contribution of NK cells is noteworthy, their effect could be included indirectly in parameter $a$ (interaction rate between tumour cells and TILs) which is a fitted parameter in our model. 
3) Interaction between tumour cells and TILs is dependent on the following biological processes:

- The interaction between tumour cells and TILs can only occur if tumour-specific antigens are processed and presented via MHC class I pathway (parameter $M H C I$ ). MHC class I needs to be expressed on the surface of tumour cells in order for T cells to recognize the target (Germain 1994). Downregulation of MHC class I is an important strategy for tumour cells in order to escape immune surveillance (Dunn et al. 2004). In our model, we only distinguish between $\mathrm{MHC}$ class I positive (MHC-I ${ }^{+}$, one or more $\mathrm{MHC}$ class I complexes are expressed on cell surface) and MHC class I negative tumour cells (MHC-I', no MHC class I complexes are expressed). Fraction of MHC-I $\mathrm{I}^{+}$tumour cells is characterized with the parameter $M H C I$.

- Tumour mutational burden is proportional to single nucleotide variations of a tumour cell line (parameter $S N V$ ).

A number of studies have suggested that the tumour mutation burden could predict the response to immunotherapy (Rizvi et al. 2015; Goodman et al. 2017). An increased tumour mutation burden leads to an elevated number of neoantigens able to trigger an immune response, a pre-determining factor for the selection of candidates for immunotherapy. We assume a linear relationship between single nucleotide variations $(\mathrm{SNV})$ in the genome of a specific tumour cell line, and the number of cell line-specific tumour antigens that could be produced and presented via MHC class I pathway, as a consequence of SNV.

- TILs can be inactivated via PD-1/PD-L1 pathway (parameters PD1, PDL1).

When tumour cells and TILs interact, we allow for two possible outcomes: (a) the tumour cell dies as a consequence of granzyme and perforin release by the TIL (Peters et al. 1991), or (b) the TIL becomes dysfunctional and dies because of the activation of the PD-1/PDL1 pathway (Dong et al. 2002). Fraction of tumour cells expressing PD-L1 and the fraction of TILs expressing PD-1 are set with parameters $P D L 1$ and $P D 1$, respectively. 

remain bound to PD-1 receptors until the natural death of T cells. Therefore the difference

- Anti-PD-1 pharmacodynamics (parameter $P D 1_{\text {occup }}$ ) is influenced by the natural death rate of TILs.

Since no experimental data about the pharmacokinetics (PK) and pharmacodynamics (PD) of murine anti-PD-1 antibodies could be found in literature, we assess the effect of PD-1 receptor blockade by referring to human data, namely to a Phase I study of the anti-PD-1 antibody nivolumab (Brahmer et al. 2010). In this study it was shown that after one application of nivolumab, more than 70\% of PD-1 receptors on circulating T cells remain occupied for more than 2 months. Our main assumption is that anti-PD-1 antibodies between the life span of human T cells and murine T cells (model parameter $d$ ) is critical, because it influences the difference between human and murine anti-PD-1 pharmacokinetics and pharmacodynamics. Based on this assumption, we take nivolumab data and scale it accordingly to the difference in half-lives of human T cells (77 days) (Hellerstein et al. 1999) versus murine T cells (41 hours) (De Boer et al. 2003). Finally, to match the assigned dose and schedule in the simulation, we interpolate the so obtained murine anti-PD-1 PK and PD. The result is parameter $P D 1_{\text {occup }}$, which describes the occupancy of PD-1 receptors on T cells at each time point of the simulation as determined by anti-PD-1 dose, which is also scaled by the ratio of human to murine body mass. This scaling from human to murine anti-PD-1 PK and PD is currently the best possible approximation for our task. If the murine anti-PD-1 PK and PD data becomes available in the future, it could be incorporated into the model easily.

\section{4) Infiltration of $T$ cells into tumour is proportional to the tumour surface area and SNV.}

Numerous studies show strong positive correlation between infiltration of $\mathrm{T}$ cells into solid tumours and favourable prognosis (Salgado et al. 2015; Badoual et al. 2013; Simpson et al. 2010; Gooden et al. 2011; Galon et al. 2006). After the activation in regional lymph nodes, T cells use blood vessels and lymphatic vessels to migrate to tumour, as well as to infiltrate the tumour mass. It is well known that established tumours in general consist of a well- 
vascularized periphery, a seminecrotic intermediate region and an avascular necrotic core (Jain 1990). Compared to the central region, tumour vasculature is denser and more abundant at the contact between the tumour and the healthy tissue, and its density tends to decrease as tumour grows (Nagy et al. 2009). We assume that tumour vasculature is one of the bottlenecks for an effective infiltration (Teng et al. 2015), and assume proportionality of $\mathrm{T}$ cell infiltration to the tumour surface area. An additional prerequisite for effective infiltration are tumour neoantigens, which can be recognized by dendritic cells and further serve as a target for $\mathrm{T}$ cells (Teng et al. 2015; Banchereau \& Steinman 1998). Based on the evidence from a study of human melanoma, showing a strong correlation between the numbers of predicted human leukocyte antigen (HLA) class I and II (i.e. human MHC class I and II) neoantigens and the number of SNV, we assume TIL infiltration is proportional to the number of SNV (Hugo et al. 2016).

\section{5) Dead tumour cell are removed with removal rate $l$.}

Since the results of our simulations are tumour growth curves, the dynamics of dead tumour cell removal needs to be taken into account. Our model does not differentiate dead cells by cell death pathways. In a clinical study on tumour volume variation during radiotherapy for head and neck and lung squamous cell carcinoma it was shown that the half-life of lethally damaged cells in humans is approximately 4 doubling times (parameter $l$ ) (Chvetsov 2013). We were not able to identify similar studies performed on mice, therefore we adopted this data for our simulations of murine tumours by calculating parameter $l$ as 4 doubling times of the B16-F10 melanoma cell line.

\section{6) Calculation of macroscopic tumour volume (parameters $\boldsymbol{r}_{c}$ and $\boldsymbol{r}_{T I L}$ ):}

275 For transitioning from a number of tumour cells to a macroscopic tumour volume, Eq. 4 is used. We are aware that in reality, tumours are not homogeneous masses composed only of tumour cells, but a heterogeneous mixture of many other cell types which constitute the tumour microenvironment. However, due to the fact that other cell types are not being modelled, we take into account only tumour cells, dead tumour cells, and TILs when 
calculating tumour volume. We assume that both tumour cells and TILs are spherical with radius $r_{c}$ and $r_{T L}$, respectively. The dynamics of dead TILs is neglected, and the model assumes that they are removed from the tumour instantly. The inclusion of dead TILs into the model would result in an additional free parameter (removal rate), but the impact on the calculated tumour volume would be negligible. Density of (dead) TILs in a tumour is usually orders of magnitude lower than that of tumour cells (observed both in in vivo experiments as well as in our simulations (Rose et al. 2018)). Additionally, the volume of a tumour cell is an order of magnitude higher than the volume of TILs. There are many ways to measure, calculate and present tumour size. In cases of experimental data reporting tumour volumes as a product of two orthogonal tumour diameters (Sanchez-Paulete et al. 2016), we compare simulated to experimental data by assuming the simulated tumour is spherical and then calculate the square of its diameter.

\subsection{Model Tuning}

Once the model was formulated, we tuned the parameters so that the model could reproduce (fit) the observed experimental results. Most model parameters were found or 295 approximated from literature, except for the parameters $k, C_{\max }, a$, and $b$, which were assessed by fitting the model to murine experimental data from literature, in which B16-F10 melanoma was treated with anti-PD-1 antibodies (Kleffel et al. 2015). The main rationale for choosing specifically experimental data by Kleffel and colleagues was that they performed experiments with anti-PD-1 ICI both on immunodeficient, as well as on wild type mice strains. Experimental

300 data (as well as the equations of our model) clearly show that the immune system has an important impact on tumour growth even if tumour is not being treated. Namely, the growth of untreated tumour in the absence of the immune system is significantly faster than the growth of untreated tumour in mice with a fully functional immune system. To assess the aforementioned parameters, we fitted the model with different boundary conditions simultaneously to the following tumour growth data: 
1. B16-F10 melanoma implanted into NSG mice (the most immunodeficient laboratory mice strain, lacking mature T cells, B cells and NK cells), which received no further therapy after the tumour implantation (NSG control group). Boundary condition: no TILs in the system, all immune-related parameters were set to 0 .

2. B16-F10 melanoma implanted into C57BL/6 mice (wild type mice with a fully functional immune system), which received no further therapy after the tumour implantation (C57BL/6 control group). Boundary condition: $P D 1_{\text {occup }}=0$ at every time-point of the simulation.

3. B16-F10 melanoma implanted into C57BL/6 mice, which received further therapy with murine anti-PD-1 antibodies (C57BL/6 anti-PD-1 group). Boundary condition: PD1 ${ }_{\text {occup }}$ $\neq 0$ (time- and dose-dependent).

In all experiments, $2 \times 10^{5}$ B16-F10 melanoma cells were injected subcutaneously into the flanks of recipient mice. In C57BL/6 anti-PD-1-treated group, anti-PD-1 antibodies were injected intraperitoneally (i.p.), $200 \mu \mathrm{g}$ per injection, which is approximately $10 \mathrm{mg} / \mathrm{kg}$, considering that

320 the body weight of C57BL/6 mice is around $20 \mathrm{~g}$. The first anti-PD-1 dose was applied one day before the injection of tumour cells, and every two days thereafter. When fitting, we set all immune-related parameters in the model to zero $(a=0, b=0)$ in the case of NSG control group. Similarly, in the case of C57BL/6 control group, the value of treatment-related parameter $P D 1_{\text {occup }}$ was set to zero $\left(P D 1_{\text {occup }}=0\right)$. All other model parameters were kept fixed. Literature-based

325 parameters, as well as the parameters obtained by fitting, are summarized in Table 1 with the corresponding references.

\subsection{Model Predictions}

When we confirmed the ability of the model to reproduce experimental results, we tested its predictive ability on two independent experimental datasets (Sanchez-Paulete et al. 2016; Scharping et al. 2017). 
In one of the series of experiments performed by Sanchez-Paulete and colleagues, $5 \times 10^{5}$ B16-OVA melanoma cells were injected subcutaneously into the right flanks of C57BL/6 mice (Sanchez-Paulete et al. 2016). The control group of mice was administered an empty plasmid (due to the additional study objectives), and the other group was administered an empty plasmid 335 concomitant with i.p. injections of anti-PD-1 antibodies on days 4, 7 and 10. The administered anti-PD-1 dose was $100 \mu \mathrm{g}$ per injection, which is an equivalent of $5 \mathrm{mg} / \mathrm{kg}$. Tumour size was measured twice weekly and calculated as the product of two orthogonal diameters.

The second set of independent data used to validate the predictive ability of our model was taken from the series of experiments by Scharping and colleagues, in which $2.5 \times 10^{5}$ B16-F10 340 melanoma cells were injected intradermally into C57BL/6 mice (Scharping et al. 2017). The antiPD-1 group was administered i.p. $200 \mu \mathrm{g} /$ injection (equivalent of $5 \mathrm{mg} / \mathrm{kg}$ ) of anti-PD-1 antibodies with concomitant injection of phosphate-buffered saline (PBS) (due to the other study objectives), starting on day 5 and every 4 days thereafter. Meanwhile, the control group was administered antiPD-1 isotype control immunoglobulin G (IgG) and PBS, according to the same schedule. Tumour 345 size was reported as the product of two orthogonal diameters.

We performed simulations with all model parameters fixed to the values summarized in Table 1, except for the boundary conditions and parameters specific for each dataset (summarized in Table 2), such as the initial number of implanted cells, and anti-PD-1 dose and schedule. Additionally, the PD-L1 expression on B16-OVA cells was found to be different from 350 the PD-L1 expression on B16-F10 cells (100\% versus $37.5 \%$, respectively, measured by flow cytometry) (Contreras-Sandoval et al. 2016). For the simulations of B16-OVA cell line, we assumed the intrinsic parameters $k, C_{\max }, a$, and $b$ to be the same as obtained by fitting the model to the B16-F10, because they both originate from the B16 cell line. There is no data in the existing literature describing differences between B16-F10 and B16-OVA proliferation rate. Besides PD-

355 L1 expression, B16-OVA most likely differs from B16-F10 only in the presence of ovalbumin, one of many tumour specific antigens (Brown et al. 2001). 


\begin{tabular}{|c|c|c|c|}
\hline & MODEL TUNING & \multicolumn{2}{|c|}{ MODEL PREDICTIONS } \\
\hline REFERENCE & (Kleffel et al. 2015) & (Sanchez-Paulete et al. 2016) & (Scharping et al. 2017) \\
\hline CELL LINE & B16-F10 & B16-OVA & B16-F10 \\
\hline VARIABLE & \multicolumn{3}{|c|}{ INITIAL VALUE } \\
\hline$C$ & $2 \times 10^{5}$ & $5 \times 10^{5}$ & $2.5 \times 10^{5}$ \\
\hline PARAMETER & \multicolumn{3}{|c|}{ VALUE } \\
\hline$P D L 1$ & $37.5 \%$ & $\begin{array}{l}100 \% \text { (Contreras-Sandoval et } \\
\text { al. 2016) }\end{array}$ & $37.5 \%$ \\
\hline$P D 1_{\text {occup }}($ dose $)$ & $200 \mu \mathrm{g}$ per injection, i.p. & $100 \mu \mathrm{g}$ per injection, i.p. & $200 \mu \mathrm{g}$ per injection, i.p. \\
\hline $\begin{array}{l}\text { PD1 } 1_{\text {occup }} \\
\text { (schedule) }\end{array}$ & $\begin{array}{l}\text { One day before implantation } \\
\text { and every two days thereafter }\end{array}$ & $\begin{array}{l}\text { Days } 4,7 \text { and } 10 \text { after } \\
\text { implantation }\end{array}$ & $\begin{array}{c}\text { Days } 5,9,13 \text {, and } 17 \text { after } \\
\text { implantation }\end{array}$ \\
\hline
\end{tabular}

Table 2: Difference in boundary conditions and model parameters, used for tuning the model and generating model predictions. All other model parameters were kept fixed (see Table 1).

In order to quantitatively assess the generated model predictions, we calculated mean relative deviation of the simulated tumour volume to the experimental data (Eq. 5).

$$
\bar{\delta}=\left(\sum_{i=1}^{N} \frac{\left|V_{s, i}-V_{e, i}\right|}{V_{e, i}}\right) / N
$$

In Eq. 5, $\bar{\delta}$ represents mean relative deviation, $V_{s}$ and $V_{e}$ represent simulated and experimentally measured tumour volume (mean value), respectively, and $i$ is an index which runs over all time points of number $N$ experimental data. We exclude the points at $\mathrm{t}=0$ from the calculations.

\subsection{Sensitivity Study}

We performed a sensitivity study to explore the impact of model parameters on the simulated results. We modulated each parameter by $\pm 1 \%, \pm 10 \%$ and $\pm 20 \%$ from the literaturebased/fitted values (Table 1) and calculated a time-averaged sensitivity coefficient (Eq. 6) as an average of absolute sensitivity coefficients at each time point of the simulation (Eq. 7) (Neumann et al. 2010).

$$
\begin{aligned}
& s_{j}=\frac{1}{N} \sum_{t=1}^{N}\left|s_{j}(t)\right| \\
& s_{j}(t)=\frac{p_{j}}{V} \frac{d V}{d p_{j}}
\end{aligned}
$$


In Eq. 6 and 7, $p_{j}$ and $s_{j}$ represent the j-th parameter and its corresponding sensitivity coefficient, respectively. $V$ represents tumour volume, and $N$ is number of time steps in the simulation. In our 375 sensitivity study, $N=1201$ (simulated time interval: 20 days, time step: $1 / 60$ day).

Because the sensitivities of the parameters depend strongly on the initial parameter values, we also performed a sensitivity study by exploring the entire possible range of individual parameters. We first assessed the meaningful range of each studied parameter and then uniformly increased its value, starting from the lower bound. If the lower bound being equal to zero resulted in no visual difference between the treated and the untreated group, we increased the lower bound to be slightly higher than zero, in order to obtain more illustrative plots. The results are presented graphically as simulated tumour growth curves. This set of sensitivity studies was performed on a longer time scale (60 days) to better visualize the difference in simulation results between the control and treatment cases. However, the model was built and tuned to simulate anti-PD-1 murine experiments, which are usually conducted on shorter time scales (15-30 days). Therefore, the late tumour responses observed in this sensitivity study should be interpreted conservatively. We labelled the unrealistic scenarios, when the murine tumour volume becomes larger than $850 \mathrm{~mm}^{3}$ (in real experiments, mice would most likely already have died or been sacrificed). This threshold was set according to the C57BL/6 control group experiments (Kleffel et al. 2015).

Finally, we performed a sensitivity study by varying all parameters simultaneously. Parameters values were drawn randomly from Gaussian distributions with mean $\mu$ equal to the corresponding value from Table 1 and the standard deviation $\sigma$ equal to $\sigma=0.1 \mu$ (first set of simulations) and $\sigma=0.5 \mu$ (second set of simulations). We performed 500 simulations for each set. The results are presented as box plots in Figure 7 . 


\section{Results}

\subsection{Model Tuning}

The model was tuned by fitting it simultaneously to experimental data of three groups of mice (Kleffel et al. 2015) (Figure 1): Immunodeficient NSG control group (blue diamonds), wild type C57BL/6 control group (green squares), and wild type anti-PD-1 group (red circles). Fitted tumour growth curves are represented with blue solid line (NSG control group), green dash-dot line (C57BL/6 control group), and red dashed line (C57BL/6 anti-PD-1 group). The obtained parameter values were: tumour growth rate $k=0.038$ day $^{-1}$, maximum number of cells in tumour $C_{\max }=1.45 \times 10^{11}$, interaction rate between tumour cells and tumour infiltrating lymphocytes $a=$ $5.64 \times 10^{-9}$ day $^{-1}$, and infiltration rate of T cells into tumour $b=7.63 \times 10^{-3}$ day $^{-1}$. All other model parameters are summarized in Table 1.

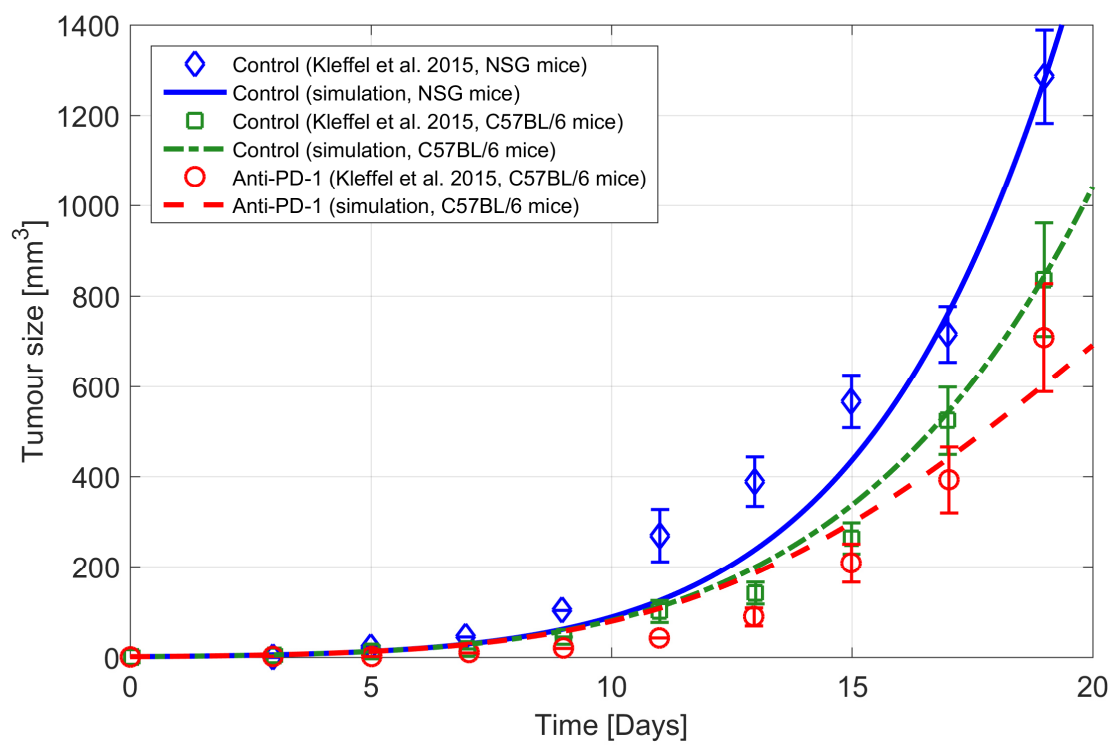

Figure 1: Model tuning. The model with free parameters $k, C_{\max }, a$, and $b$ was fitted to experimental data (Kleffel et al. 2015), in which $2 \times 10^{5}$ B16-F10 melanoma cells were injected into three groups of mice. Blue diamonds: immunodeficient NSG control group, which received no further therapy after the tumour implantation. Green squares: wild type C57BL/6 control group, which received no further therapy after the tumour implantation. Red squares: wild type C57BL/6 control group, treated with anti-PD-1 antibodies (200 $\mu$ g/injection, every two days). The experimental data is represented as mean $\pm \mathrm{SD}$. The fitted simulations are represented with lines. Blue solid line: NSG control group. Green dash-dot line: C57BL/6 control group. Red dashed line: C57BL/6 anti-PD-1 group. 


\subsection{Model Predictions}

We assessed the predictive ability of the model by using two independent experimental 410 datasets from literature with some of the initial conditions (parameters) being different but wellknown (Table 2), and compared the data to our simulations. First, we simulated experimental scenarios of Sanchez-Paulete and colleagues. As seen in Figure 2, the simulated growth curves are in good agreement with the experimental data. The model slightly underestimates the growth of the untreated tumour. Mean relative deviation of simulated control tumour volume (solid line green) to the mean value of experimental data (green squares) is $13 \%$. On the other hand, the model slightly overestimates the growth of anti-PD-1-treated tumour. Mean relative deviation of simulated anti-PD-1-treated tumour volume (dashed line - red) to the mean value of experimental data (red circles) is $19 \%$.

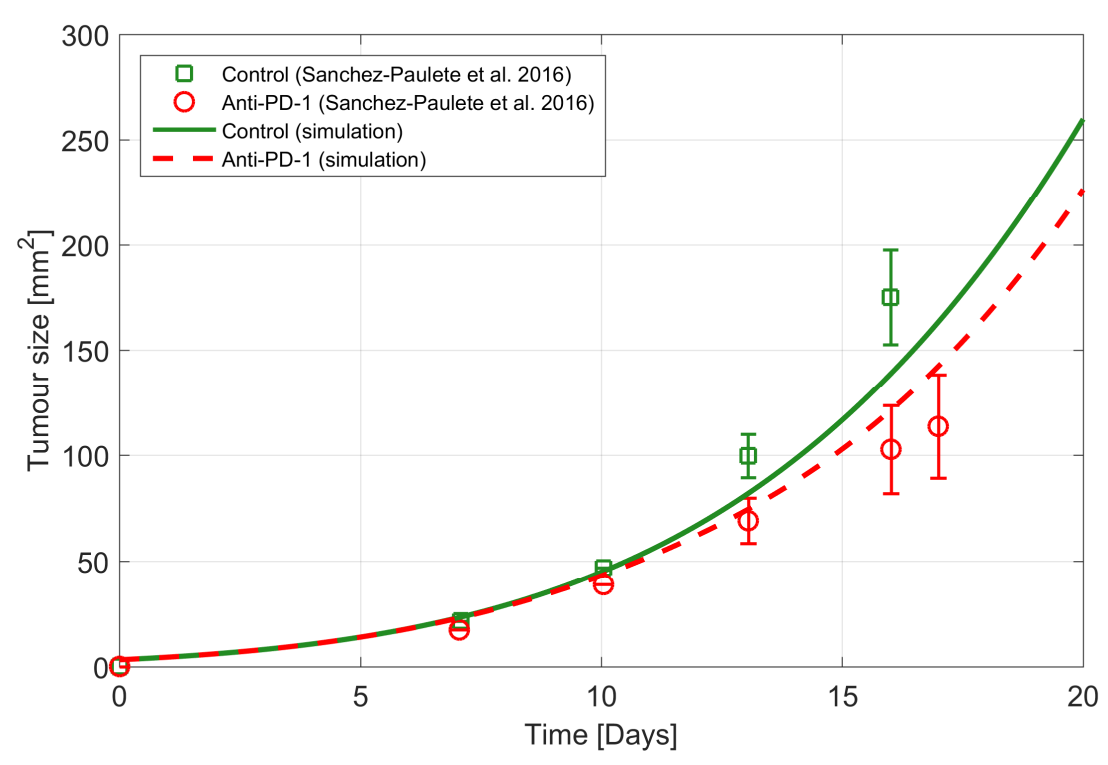

Figure 2: Model predictions versus experimental data (Sanchez-Paulete et al. 2016). Model predictions were compared to experimental data, in which $5 \times 10^{5}$ B16-OVA melanoma cells were injected subcutaneously into the flanks of C57BL/6 mice and treated with anti-PD-1 antibodies. Green squares: control group. Red circles: anti-PD-1 group (100 $\mu \mathrm{g}$ /injection, days 4, 7 and 10). The experimental data is represented as mean $\pm \mathrm{SD}$. Simulations are represented with lines. Green solid line: control group. Red dashed line: anti-PD-1 group.

The second experimental dataset that we used to test the predictive ability of our model was from Scharping and colleagues. Agreement between the simulations and the experimental 
data is presented in Figure 3. Also in this case, the experimental data is in good agreement with our simulations. Mean relative deviation of simulated control tumour volume (Figure 3, left: solid line - green) to the mean value of experimental data (Figure 3, left: green squares) is $14 \%$, while 425 mean relative deviation of simulated anti-PD-1-treated tumour volume (Figure 3, right: solid line - red) to the mean value of experimental data (Figure 3, right: red circles) is $20 \%$.
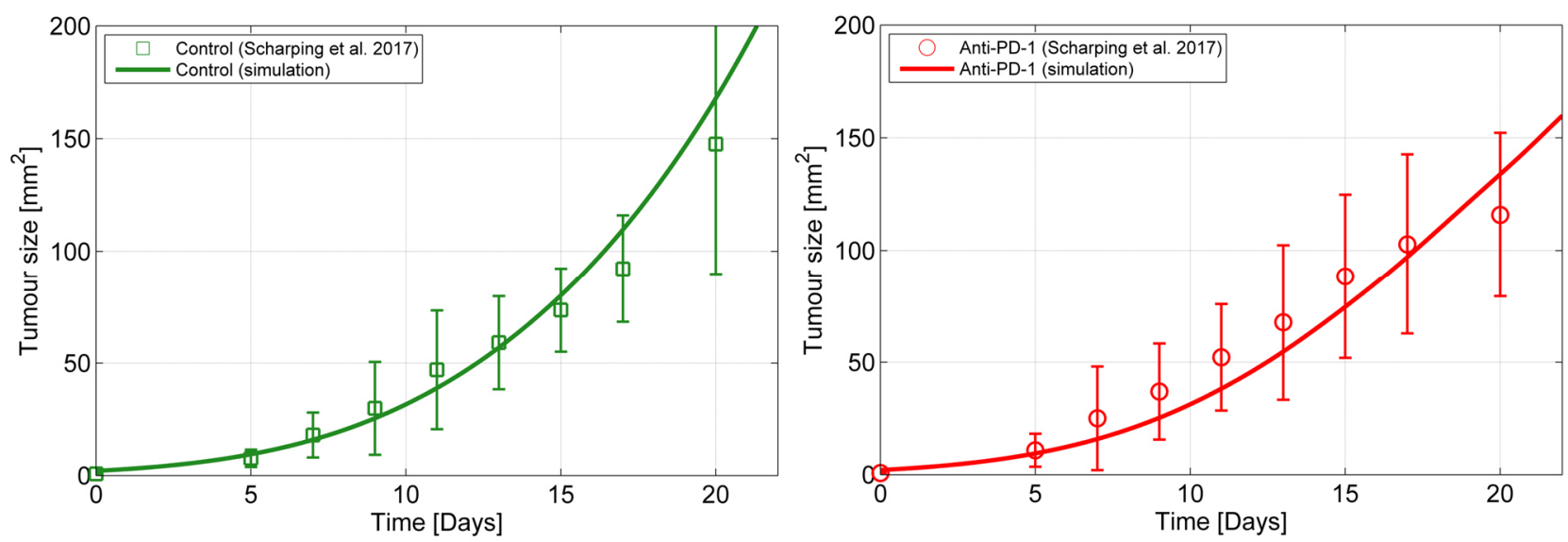

Figure 3: Model predictions versus experimental data (Scharping et al. 2017). Model predictions were compared to experimental data, in which $2.5 \times 10^{5} \mathrm{~B} 16-\mathrm{F} 10$ melanoma cells were injected intradermally into the flanks of C57BL/6 mice and treated with anti-PD-1 antibodies. The experimental data is represented as mean \pm SD. Simulations are represented with solid lines. Left: control group. Right: anti-PD-1 group (200 $\mu \mathrm{g} /$ injection, days 5, 9, 13, and 17).

\subsection{Sensitivity Study}

To test robustness of the model and to study the impact of model parameters on simulation results, we performed a sensitivity study by calculating time-averaged sensitivity coefficients for 430 each model parameter. We modulated the parameter values, summarized in Table 1 , by $\pm 1 \%$, $\pm 10 \%$ and $\pm 20 \%$. The results for the $+10 \%$ scenario are shown in Figure 4 , while other scenarios $( \pm 1 \%, \pm 20 \%)$ lead to similar trends. In case of untreated control tumours, by far the most sensitive parameter was tumour growth rate $k$ with a sensitivity coefficient more than an order of magnitude higher that of other parameters, except tumour cell volume $V_{c}\left(=\frac{4}{3} \pi r_{c}^{3}\right)$ with the expected value 435 around 1 (which could be easily derived from Eq. 4 by taking into account that $V_{c}>V_{T I L}$ ). Maximum number of cells in tumour $C_{\max }$ appeared to be the third most sensitive parameter, which supplements the group of the intrinsic tumour parameters $\left(k, V_{c}, C_{\max }\right)$, having the highest relative 
sensitivity coefficients. Among other parameters, the most sensitive were $S N V, b, M H C I$, and $a$, in order of decreasing sensitivity. The remaining five parameters ( $\left.P D L 1, P D 1, d, l, V_{T I L}, P D 1_{\text {occup }}\right)$

440 were the least sensitive. Sensitivity analysis of anti-PD-1 treated tumour simulations did not show notably different picture. Sensitivity coefficient values of the intrinsic tumour parameters $\left(k, V_{c}\right.$, $C_{\max }$ ) were slightly lower in the case of treatment with anti-PD-1, while sensitivity coefficients of the majority of the other parameters increased slightly $\left(S N V, b, a, M H C I, d, l, V_{T I L}, P D 1_{\text {occup }}\right)$. Surprisingly, relative sensitivity coefficients of parameters $P D L 1$ and $P D 1$ slightly decreased. The 445 reason is that the number of immune-suppressive PD-1/PD-L1 interactions between tumour cells and TILs is largely reduced under anti-PD-1 therapy. Consequently, the modulation of parameters $P D L 1$ and $P D 1$ has less impact on the resulting tumour growth under treatment as compared to control.

Although relative sensitivity coefficients provide insight into the relationship between parameters and simulation results to some extent, their values strongly depend on their initial values. For instance, MHC class I expression $M H C I$ was $2.3 \%$ in our case, and by modulating this
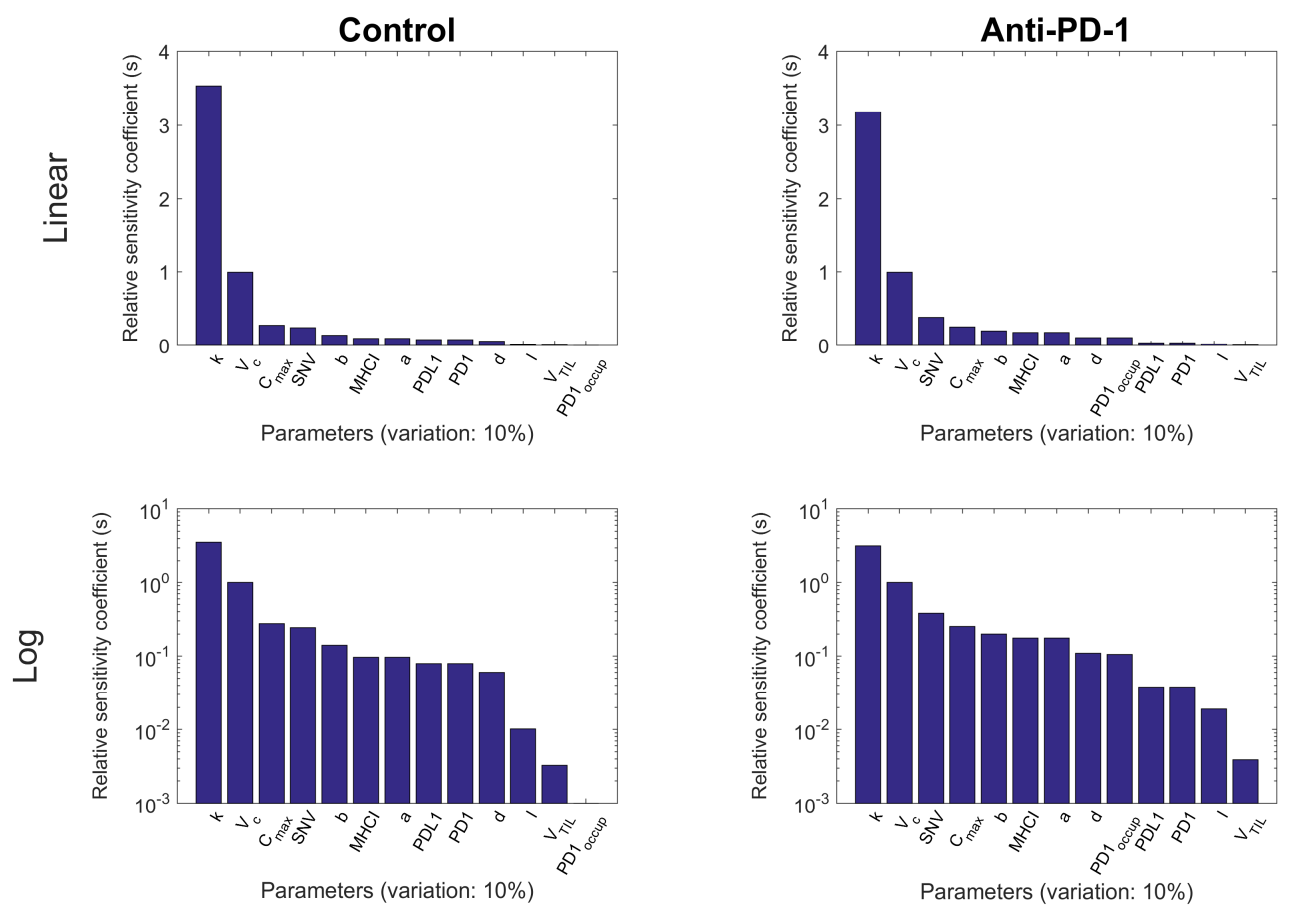

Figure 4: Sensitivity study - time-averaged sensitivity coefficients. Left - control tumour simulations sensitivity coefficients, right - anti-PD-1-treated tumour simulations sensitivity coefficients. Top - linear scale, bottom logarithmic scale. 
parameter $\pm 10 \%$ of the initial value, we investigated just a tiny space of all possible MHC class I values $(2.07 \%-2.53 \%)$. Therefore, we also performed a sensitivity study by modulating the parameters through their entire expected and meaningful range. We focused mainly on the parameters which have a known range or which could be relatively easily measured experimentally, namely $k, S N V, M H C I$ and $P D L 1$. The results are presented graphically in Figure

5.

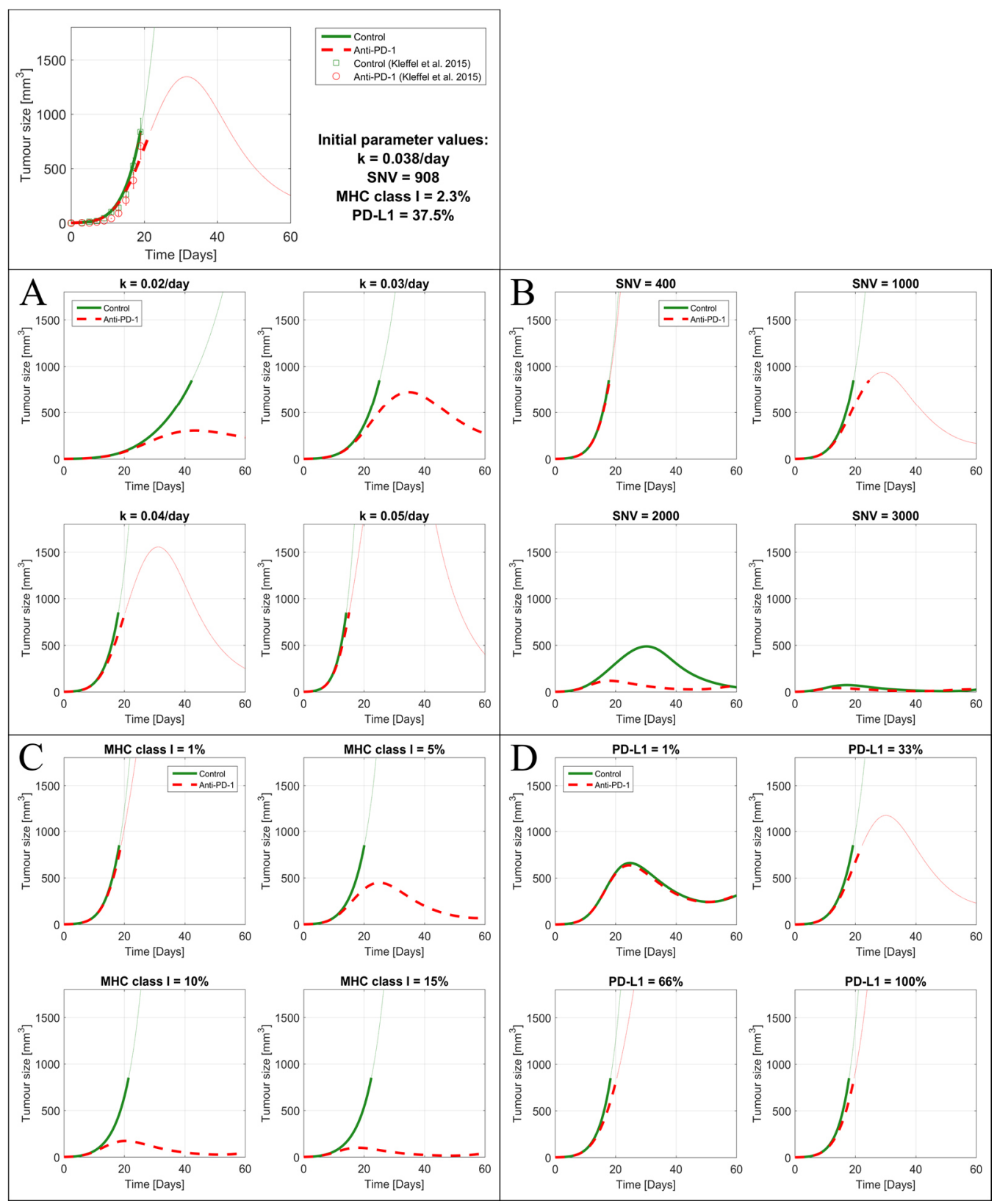

Figure 5: Sensitivity study - modulation of absolute values of parameters through the entire possible range. Top left: reference results - simulations performed with parameter values from Table 1, (A) $k$ sensitivity, (B) SNV sensitivity, (C) MHCI sensitivity, (D) PDL1 sensitivity. Thick green solid line (control) and thick red dashed line (anti-PD-1) represent simulation results, which could be observed in real experiments. Thin dotted lines represent unrealistic scenarios for murine tumours $\left(\mathrm{V}>850 \mathrm{~mm}^{3}\right.$, mice dead or sacrificed). 
As already indicated by sensitivity coefficients, tumour growth rate $k$ has a significant effect on the model results (Figure 5A). However, with the chosen set of model parameters, the 460 simulated growth of control tumour is not significantly different from the anti-PD-1-treated tumour for all $k$ values (for the realistic scenarios). The model suggests that late responses to antiPD-1 immunotherapy might be observed in case of less aggressive tumours with longer cell cycle time (lower $k$ ). A similar trend is observed with single nucleotide variations $S N V$ (Figure 5B). On the other hand, MHC class I expression $M H C I$ seems to play an important role in discriminating 465 responders from non-responders to anti-PD-1. In our simulations, tumours with low MHC class I expression appear resistant to anti-PD-1 therapy, while tumours with higher MHC class I expression achieve favourable responses early after the treatment initiation (Figure 5C). Surprisingly, PD-L1 expression does not have a significant impact on treatment results, if the simulations are performed with the set of parameters summarized in Table 1 (Figure 5D).

Keeping in mind that positive PD-L1 expression is usually a key criteria for treatment with anti-PD-1 antibodies in clinics, and is often connected with favourable outcome (Reck et al. 2016; Gandini et al. 2016), we tested whether our model is insensitive to PD-L1 expression in general or just as a consequence of the chosen set of model parameters. We performed another PD-L1 sensitivity study, this time with an increased MHC class I expression $(M H C I=10 \%)$. In

475 this scenario, the model does become sensitive to PD-L1 (Figure 6). Based on its expression, it is now possible to discriminate responders from non-responders to anti-PD-1 antibodies. Interestingly, the model indicates that the best response could be observed for moderate PDL1 values $(P D L 1=33 \%$ and $66 \%$, while the growth of tumour with $100 \%$ PD-L1 expression is only delayed, comparing to the control group. 

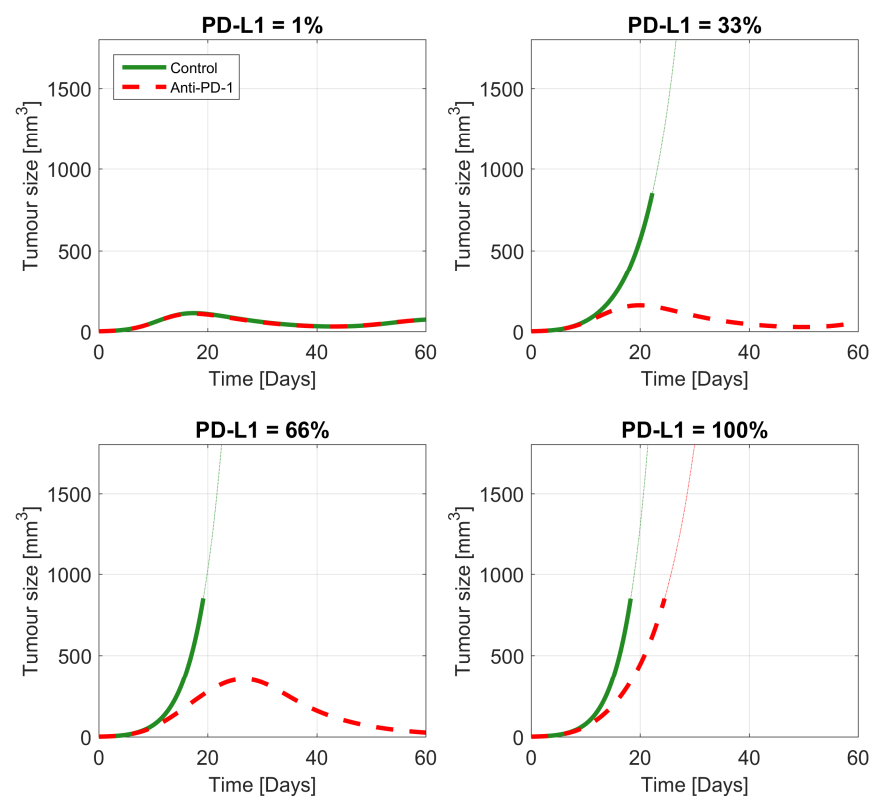

Figure 6: PD-L1 sensitivity study with different set of parameters as compared to Table 1. MHC class I expression was increased to $M H C I=10 \%$. Thick green solid line (control) and thick red dashed line (anti-PD-1) represent simulation results, which could be observed in real experiments. Thin dotted lines represent unrealistic scenarios for murine tumours $\left(\mathrm{V}>850 \mathrm{~mm}^{3}\right.$, mice dead or sacrificed).

Finally, we performed sensitivity studies by releasing all parameters simultaneously. Parameter values were drawn randomly from Gaussian distributions. In the first set of simulations $(\mu, \sigma=0.1 \mu)$ (Figure 7A), all control (untreated) tumours escaped the immune system and grew above $850 \mathrm{~mm}^{3}$ by day 30 . Meanwhile, the median of tumours treated with anti-PD-1 remained resistant to therapy. The best observed response to anti-PD-1 was a delay in the tumour growth with the minimum tumour volume $V=255 \mathrm{~mm}^{3}$ on day 30 . All outliers were found above the median (except on day 0). When we increased the standard deviation to $\sigma=0.5 \mu$ (second set of simulations), the results became far more heterogeneous (Figure 7B). The medians of both groups slightly decreased. Both non-responsive tumours as well as complete responders were observed in the anti-PD-1 group on day 30. Even in the control group, the immune system was able to eradicate tumour for some sets of parameters. All outliers were again above the median. 


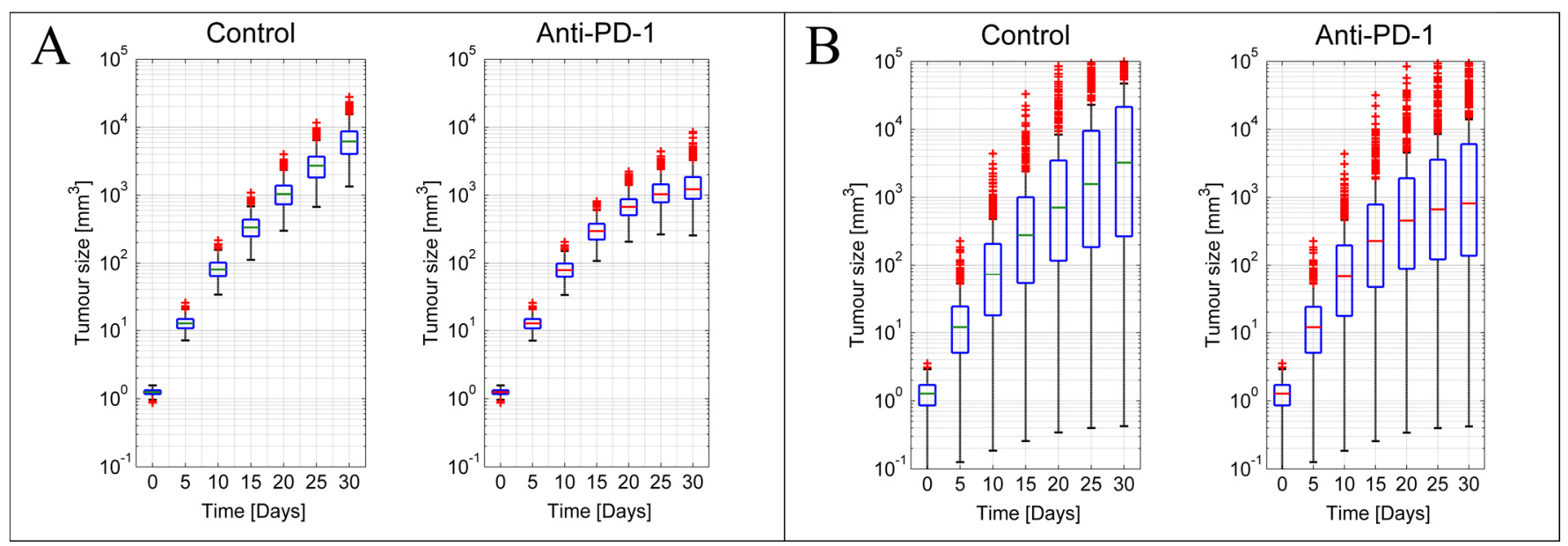

Figure 7: Sensitivity study - all parameters released simultaneously. Parameter values were drawn randomly from Gaussian distributions with mean $\mu$ equal to the corresponding value from Table 1 and the standard deviation $\sigma$ equal to $\sigma=0.1 \mu($ Figure $7 \mathrm{~A})$ and $\sigma=$ $0.5 \mu$ (Figure 7B). 500 simulations for each set were performed. The results are represented as standard box plots, in which the central mark indicates the median, the top and bottom box edges indicate the $75^{\text {th }}$ and $25^{\text {th }}$ percentiles, respectively. The whiskers represent the extreme data points excluding outliers. The outliers are plotted as the '+' symbols. 


\section{Discussion}

We have developed a deterministic population model, based on a bottom-up approach, which is able to reproduce and predict tumour response to therapy with anti-PD-1 antibodies. We 500 have built a model with the fewest possible number of parameters without losing predictive power. Out of 13 model parameters, only 4 of them are free (tumour growth rate $k$, maximum number of cells in tumour $C_{\max }$, interaction rate between tumour cells and tumour infiltrating lymphocytes $a$, and infiltration rate of $\mathrm{T}$ cells into tumour $b$ ), while all other parameters were estimated from literature. Importantly, all of the free parameters could be measured experimentally. For example, $k$ and $C_{\max }$ characterize intrinsic tumour growth in the absence of immune system, and could be measured by implantation of tumour cells into immune-deficient mice strains, such as nude mice, NSG mice, or other immune-deficient model organisms. From experimental data in Figure 1 it is obvious that the control tumours implanted into NSG mice grow much faster compared to the control tumours implanted into C57BL/6 mice with a fully functional immune system. Assessment

510 of the parameter $a$ could be done in vitro by a cytotoxicity assay (tumour cells and $\mathrm{T}$ cells) followed by cytometric quantification of cell death. It might also be possible to assess parameter $b$ - first by implanting tumour cells into PD-1 knockout mice (to assure that TILs are not being inactivated via PD-1/PD-L1 pathway once they infiltrate tumour), and then cytometrically determine the TILs density in tumour versus tumour surface area at various time points of tumour 515 growth.

When we tuned the model on data from literature (Kleffel et al. 2015), we fitted the parameters $k, C_{\max }, a$, and $b$ simultaneously to three experiments (NSG control group, C57BL/6 control group, C57BL anti-PD-1 group) (Figure 1). The reason is that only averaged tumour growth data was available. Namely, in Figure 1 it could be seen that the averaged experimental 520 data of NSG control group follows Gompertz function, while the averaged experimental data of C57BL/6 control and anti-PD-1 group is nearly exponential. This may or may not be true for the growth of each individual tumour. However, it was impossible to assess the parameters $k$ and $C_{\max }$ separately by fitting the model exclusively to NSG control group and further obtain good fits on 
the other two experiments (C57BL/6 control group, C57BL anti-PD-1 group). To properly 525 measure model parameters in the future, experimental data should be analysed by taking into account high inter-patient variability, for instance by fitting the model to individual tumour growth curves using non-linear mixed effects models (Lindstrom \& Bates 1990). Especially in immunotherapy, inter-patient variability for the same kind of treatment could be substantial, ranging from complete response (CR) to progressive disease (PD) (Dovedi et al. 2017), which 530 consequently reduces the informative value of averaged tumour data for quantitative studies. Furthermore, our model was tuned to simulate melanomas (B16-F10, B16-OVA). The model can easily be used to simulate other types of cancer, but the free and fixed parameters should be tuned and measured separately for each individual tumour location and histopathology. For example, different types of tumours can vary significantly in intrinsic growth rate $k$, SNV, MHC class I, and PD-L1 expression (Castle et al. 2015). Additionally, because of different vascular density and tumour microenvironment composition (containing different amounts of immune-suppressive cells, e.g. regulatory $\mathrm{T}$ cells (Tregs), myeloid-derived suppressor cells (MDSCs), tumourassociated macrophages (TAMs)), the (macro)parameters $a$ and $b$ can vary significantly between different types of tumours (Lindau et al. 2013; Mei et al. 2016). Model predictions, performed on independent experimental data are within expectations (Figures 2 and 3) and largely within experimental uncertainties. The model is able to predict the growth of control, as well as anti-PD-1 treated tumours adequately well in experiments with different types of melanoma (B16-F10 vs. B16-OVA), PD-L1 expression, number of implanted tumour cells, and different anti-PD-1 dose and schedule (Sanchez-Paulete et al. 2016; Scharping 545 et al. 2017). Since the majority of model parameters were taken and assessed from various published studies and were not specifically measured in every particular experiment that we simulated, the agreement between the simulations and the experimental data is even more surprising. Furthermore, in our model all parameters (except $P D 1_{\text {occup }}$ ) are assumed to be constant over the course of the simulation, which might not be true in reality. For instance, studies report that PD-L1 could be upregulated as a response to interferon gamma (IFN $\gamma$ ) released by TILs, representing an adaptive immune-resistance mechanism, or as a consequence of oncogenic 
signalling pathways, representing an intrinsic immune-resistance mechanism (Pardoll 2012; Parsa et al. 2007; Atefi et al. 2014). Furthermore, the number of single nucleotide variations (SNVs) in the genome of tumour cells is assumed to be constant during tumour progression and anti-PD-1 therapy. Contrarily, in a study by Efremova and colleagues it was shown that anti-PD-1/PD-L1 immunotherapy potentiates immunoediting, which can result in alterations to the tumour genome, the neoantigen landscape, and the tumour heterogeneity. However, the strongest effect of immunoediting was observed in hypermutated tumours (MC38 colon carcinoma), whereas the immunoediting effects were less pronounced in non-hypermutated tumours (CT26 colon 560 carcinoma) (Efremova et al. 2018). For the case of B16 melanoma, which harbors 3-fold fewer SNVs compared to CT26 (Castle et al. 2015), our assumption of keeping SNV constant during simulations is most likely a good approximation. However, the immunoediting effects should be incorporated into the model for simulations of hypermutated tumours. Another important parameter with likely dynamic nature is MHC class I expression. Downregulation of the MHC 565 class I pathway is one of the most important strategies tumours use to escape immune surveillance. MHC class I downregulation could be reversible or non-reversible and further connected with the stage of tumour development (Smahel 2017). Presumably, at an early stage, the majority of tumours are MHC class I positive, turning into a MHC class I negative phenotype at a later stage due to T cell-mediated immune selection (Garrido et al. 2017). If MHC class I downregulation is reversible, its function can be restored as a response to different cytokines, for instance as a response to IFN $\gamma$ (Zhou 2009). However, until the dynamics of these parameters are determined and measured experimentally, we treat them as second-order corrections and do not include them in our model.

The results of sensitivity studies (Figures 4 and 5A) reveal that the model is very sensitive 575 to intrinsic growth rate $k$, which is expected. However, since $k$ describes uncontrolled sustained proliferation (Hanahan \& Weinberg 2000; Hanahan \& Weinberg 2011) in the absence of an immune response and is most likely connected with the cell cycle of specific tumour cell lines (Malumbres \& Barbacid 2009), modulation of $k$ only results in the difference in time a tumour needs to grow to a certain volume, regardless of whether or not is being treated with ICI. As seen 
580 in Figure 5A, the growth of anti-PD-1-treated tumour does not differ notably from the growth of untreated tumour in the first 20 days, regardless of the value of $k$. Therefore, according to our model, it is not possible to predict early response to ICI based on $k$ value. The model does suggest late tumour responses to ICI for less aggressive tumours with lower $k$, however, these finding are questionable, because the model was tuned on experiments conducted on short time scales of 0 58520 days.

Similar behaviour is also observed when performing a sensitivity study of the parameter single nucleotide variations $S N V$. More immunogenic tumours with higher mutational load grow slower, or even fail to establish, compared to less immunogenic tumours with lower mutational load (Figures 4 and 5B). Alternatively, the value of $S N V$ does not discriminate responders from non-responders to anti-PD-1 therapy - the difference in growth curves between treated and untreated group is negligible for all $S N V$ values, for the parameter set in Table 1. This result is consistent with experimental observations in human melanoma patients reporting that high mutational load does not predict response to anti-PD-1 therapy (Hugo et al. 2016). Although the median $S N V$ of responders was higher than the median $S N V$ of non-responders in this study, the 595 difference was not statistically significant $(\mathrm{p}=0.30)$. However, Hugo et al. did show that mutational load was found to predict clinical benefit as reflected by overall survival (OS). The top third of patients by $S N V$ value had longer OS compared to the bottom third of patients $(\mathrm{p}=0.005)$. Of note, the same trend, although not statistically significant, was also observed in patients not treated with anti-PD-1 immunotherapy. These findings are in accordance with the results of our

600 sensitivity study, where we have shown that progression of tumours with higher $S N V$ is much slower compared to tumours with low mutational burden, regardless of whether they are being treated or not.

As we explained in the sensitivity study, the insensitivity of the model to PD-L1 expression seems surprising and contradictory. PD-L1 expression on tumour cells is usually 605 considered to be a favourable prognostic biomarker of response to anti-PD-1 therapies (Reck et al. 2016; Gandini et al. 2016), although a proportion of patients with PD-L1 negative tumours also 
respond to such therapy (Taube 2014). Additionally, especially in the case of melanoma, it is also well known that the results of pre-clinical anti-PD-1 trials do not always correlate with the results observed in clinical settings. While anti-PD-1, as well as anti-CTLA-4 immunotherapies represent a major breakthrough for human melanoma patients, resulting in high response rates and favourable outcomes compared to other systemic therapies (Hodi et al. 2010; Ribas et al. 2015), pre-clinical melanomas are mostly insensitive to immune checkpoint inhibitors (ICI) as monotherapies (Kleffel et al. 2015; Sanchez-Paulete et al. 2016; Scharping et al. 2017). The latter is in accordance with observations of our PD-L1 sensitivity study, where the simulated tumour 615 remains resistant to anti-PD-L1 therapy for all values of parameter $P D L 1$ (1-100\%) (Figure 5D). In our model, this tumour response behaviour is mainly due to very low MHC class I expression (2.3\%) of B16 melanoma cell line (Yamamura et al. 2015). If we tried to mimic human tumour properties and slightly increase the fraction of tumour cells expressing MHC class I, e.g. increase MHCI from $2.3 \%$ to $10 \%$, the model does become more sensitive to anti-PD-1 therapy (Figure 6).

620 This might explain why a proportion of human melanoma patients, in contrast with pre-clinical models, achieve favourable and durable responses to anti-PD-1 immunotherapy - studies have shown that MHC class I expression in human melanoma tumours can vary significantly among patients with values from $0-100 \%$ (Ott et al. 2017). Furthermore, the simulations with increased MHCI (10\%) suggest that the best tumour response to anti-PD-1 should be observed for moderate $P D L 1$ values $(P D L 1=33 \%$ and $66 \%)$, which is interesting. An explanation for this might be that while the PD-1/PD-L1 pathway protects the tumour against the T cells, the PD-1/PD-L1 pathway is also the main target for anti-PD-1 immunotherapy. Therefore, if PD-L1 expression is too high, the immune system becomes incapable of controlling the tumour in spite of PD-1/PD-L1 blockade (studies showed that PD-1 receptors on T cells are never 100\% occupied by anti-PD-1 antibodies (Brahmer et al. 2010)). On the contrary, if PD-L1 expression is too low, treating the patient with anti-PD-1 immunotherapy would not be expected to be effective because the main target is missing.

We showed in the sensitivity studies that sufficient MHC class I expression on tumour cells is one of the prerequisites for a successful immunotherapy with ICI and could serve as a 
predictive biomarker of response. Namely, T cells are able to recognize only those tumour cells, 635 which present their antigens through MHC class I receptor (Germain 1994). In the case of ineffective antigen presentation through MHC class I complex, the adaptive immune system is unable to recognize the target to attack in spite of blocking immunosuppressive PD-1/PD-L1 pathway with ICI. Indeed, in our sensitivity study, the parameter $M H C I$ was the only parameter able to discriminate responders from non-responders to anti-PD-1 therapy (Figure 5C). In favour 640 of our hypothesis, studies have shown positive correlations between defects in antigen presentation through MHC class I due to mutations in gene encoding beta-2 microglobulin $(\beta 2 \mathrm{M})$, a constitutive part of MHC class I receptor, and acquired resistance to anti-PD-1 antibodies in various human cancer types, including melanoma, non-small cell lung cancer (NSCLC) and colon cancer (Zaretsky et al. 2016; Gettinger et al. 2017; Le et al. 2017; Sade-Feldman et al. 2017).

645 However, due to high inter-patient, intra-patient, and tumour heterogeneity in different biological properties a combination of different biomarkers would most likely be superior to single biomarkers in predicting response to ICI. Based on our modelling results we speculate that a combination of MHC class I, PD-L1 and SNV might play a key role in driving response to immunotherapies, as these biomarkers provide concomitant information about tumour 650 immunogenicity (SNV), tumour ability to present antigens (MHC class I), and tumour ability to suppress immune response (PD-L1). Studies show that all three of these parameters can be highly variable among human patients (Hugo et al. 2016; Ott et al. 2017; Sunshine et al. 2017).

The main limitation of the proposed model is possible oversimplification in describing the immense complexity of the immune system. However, more complex models with even more free 655 parameters would not serve our main purpose: to build a bottom-up model with translational relevance. Although more complex models would theoretically provide a better description of the reality, their predictive ability would most likely be reduced by parameters that would not be measured experimentally. Even in our simplified model we observe that different combinations of initial inputs can lead to similar simulation results (Figure 7), and such overfitting would just be 660 potentiated in more complex models with too many free parameters. Another limitation is that we used published data to tune the model and to test its predictive ability. Parameters were not 
evaluated in these particular studies, but assessed in separate studies. Experimental verification with concurrent on-site measurements of all relevant model parameters, which is our future plan, would provide even better assessment of the predictive ability of our model. This will also allow 665 us to evaluate our model beyond murine melanoma.

\section{Conclusion}

The computational model that we have developed in this work is able to reproduce the results of anti-PD-1 pre-clinical studies, as well as to predict the results of independent studies.

670 The results of our sensitivity studies indicate that tumour major histocompatibility complex (MHC) class I expression shows promise as a predictive biomarker of response to anti-PD-1 therapy, especially when used in combination with known PD-L1 expression and information about mutational burden, such as single nucleotide variations (SNV). Additionally, our model predicts the best response to anti-PD-1 therapy for subjects with moderate PD-L1 values. 


\section{Acknowledgements}

The authors acknowledge the financial support from the Slovenian Research Agency (research core funding No. P1-0389). Thanks to Daniel Huff and Matthew Scarpelli from the University of Wisconsin - Madison for valuable comments and proofreading the manuscript.

680

\section{References}

Agur, Z. et al., 2016. Employing dynamical computational models for personalizing cancer immunotherapy. Expert opinion on biological therapy, 16(11), pp.1373-1385. Available at: https://www.tandfonline.com/doi/full/10.1080/14712598.2016.1223622 [Accessed November 27, 2017].

Atefi, M. et al., 2014. Effects of MAPK and PI3K pathways on PD-L1 expression in melanoma. Clinical cancer research : an official journal of the American Association for Cancer Research, 20(13), pp.3446-3457.

Badoual, C. et al., 2013. PD-1-expressing tumor-infiltrating T cells are a favorable prognostic

690 biomarker in HPV-associated head and neck cancer. Cancer research, 73(1), pp.128-38. Available at: http://www.ncbi.nlm.nih.gov/pubmed/23135914 [Accessed September 21, 2017].

Banchereau, J. \& Steinman, R.M., 1998. Dendritic cells and the control of immunity. Nature, 392(6673), pp.245-252. Available at: http://dx.doi.org/10.1038/32588.

695 Benzekry, S. et al., 2014. Classical Mathematical Models for Description and Prediction of Experimental Tumor Growth F. Mac Gabhann, ed. PLoS Computational Biology, 10(8), p.e1003800. Available at: http://dx.plos.org/10.1371/journal.pcbi.1003800 [Accessed September 19, 2017].

700

De Boer, R.J., Homann, D. \& Perelson, A.S., 2003. Different dynamics of CD4+ and CD8+ T cell responses during and after acute lymphocytic choriomeningitis virus infection. Journal of immunology (Baltimore, Md. : 1950), 171(8), pp.3928-35. Available at: http://www.ncbi.nlm.nih.gov/pubmed/14530309 [Accessed September 15, 2017].

Brahmer, J.R. et al., 2010. Phase I Study of Single-Agent Anti-Programmed Death-1 (MDX1106) in Refractory Solid Tumors: Safety, Clinical Activity, Pharmacodynamics, and

705 Immunologic Correlates. Journal of Clinical Oncology, 28(19), pp.3167-3175. Available at: http://www.ncbi.nlm.nih.gov/pubmed/20516446 [Accessed September 15, 2017].

Brown, D.M. et al., 2001. Tumours can act as adjuvants for humoral immunity. Immunology, 102(4), pp.486-97. Available at: http://www.ncbi.nlm.nih.gov/pubmed/11328383 [Accessed October 26, 2018].

710 Castle, J.C. et al., 2015. Mutated tumor alleles are expressed according to their DNA frequency. Scientific Reports, 4(1), p.4743. Available at: http://www.ncbi.nlm.nih.gov/pubmed/24752137 [Accessed September 15, 2017].

Chvetsov, A. V., 2013. Tumor response parameters for head and neck cancer derived from tumor-volume variation during radiation therapy. Medical Physics, 40(3), p.034101. 715 Available at: http://www.ncbi.nlm.nih.gov/pubmed/23464345 [Accessed September 15, 
2017].

Contreras-Sandoval, A.M. et al., 2016. Correlation between anti-PD-L1 tumor concentrations and tumor-specific and nonspecific biomarkers in a melanoma mouse model. Oncotarget, 7(47), pp.76891-76901. Available at: http://www.ncbi.nlm.nih.gov/pubmed/27764774 [Accessed October 9, 2017].

Cristescu, R. et al., 2018. Pan-tumor genomic biomarkers for PD-1 checkpoint blockade-based immunotherapy. Science, 362(6411), p.eaar3593. Available at: http://www.ncbi.nlm.nih.gov/pubmed/30309915 [Accessed October 18, 2018].

dePillis, L.G., Eladdadi, A. \& Radunskaya, A.E., 2014. Modeling cancer-immune responses to therapy. Journal of Pharmacokinetics and Pharmacodynamics, 41(5), pp.461-478. Available at: http://www.ncbi.nlm.nih.gov/pubmed/25281420 [Accessed November 28, 2017].

Dong, H. et al., 2002. Tumor-associated B7-H1 promotes T-cell apoptosis: A potential mechanism of immune evasion. Nature Medicine, 8(8), pp.793-800. Available at: http://www.ncbi.nlm.nih.gov/pubmed/12091876 [Accessed September 25, 2017].

Dovedi, S.J. et al., 2017. Fractionated Radiation Therapy Stimulates Antitumor Immunity Mediated by Both Resident and Infiltrating Polyclonal T-cell Populations when Combined with PD-1 Blockade. Clinical cancer research : an official journal of the American Association for Cancer Research, 23(18), pp.5514-5526. Available at:

735 http://www.ncbi.nlm.nih.gov/pubmed/28533222 [Accessed April 25, 2018].

Dunn, G.P., Old, L.J. \& Schreiber, R.D., 2004. The Three Es of Cancer Immunoediting. Annual Review of Immunology, 22(1), pp.329-360. Available at: http://www.ncbi.nlm.nih.gov/pubmed/15032581 [Accessed September 25, 2017].

Efremova, M. et al., 2018. Targeting immune checkpoints potentiates immunoediting and changes the dynamics of tumor evolution. Nature Communications, 9(1), p.32. Available at: http://www.ncbi.nlm.nih.gov/pubmed/29296022 [Accessed October 25, 2018].

Gakamsky, D.M. et al., 2005. CD8 Kinetically Promotes Ligand Binding to the T-Cell Antigen Receptor. Biophysical Journal, 89(3), pp.2121-2133. Available at: http://www.ncbi.nlm.nih.gov/pubmed/15980174 [Accessed December 11, 2017].

745 Galon, J. et al., 2006. Type, density, and location of immune cells within human colorectal tumors predict clinical outcome. Science (New York, N.Y.), 313(5795), pp.1960-4. Available at: http://www.ncbi.nlm.nih.gov/pubmed/17008531 [Accessed November 15, 2016].

Gandini, S., Massi, D. \& Mandalà, M., 2016. PD-L1 expression in cancer patients receiving anti PD-1/PD-L1 antibodies: A systematic review and meta-analysis. Critical reviews in oncology/hematology, 100, pp.88-98. Available at: http://linkinghub.elsevier.com/retrieve/pii/S1040842816300257 [Accessed November 17, 2017].

Garon, E.B. et al., 2015. Pembrolizumab for the Treatment of Non-Small-Cell Lung Cancer. New England Journal of Medicine, 372(21), pp.2018-2028. Available at: http://www.nejm.org/doi/10.1056/NEJMoa1501824 [Accessed December 2, 2016].

Garrido, F., Ruiz-Cabello, F. \& Aptsiauri, N., 2017. Rejection versus escape: the tumor MHC dilemma. Cancer immunology, immunotherapy: CII, 66(2), pp.259-271.

Germain, R.N., 1994. MHC-dependent antigen processing and peptide presentation: providing 
760 ligands for T lymphocyte activation. Cell, 76(2), pp.287-99. Available at: http://www.ncbi.nlm.nih.gov/pubmed/8293464 [Accessed September 25, 2017].

Gettinger, S. et al., 2017. Impaired HLA Class I Antigen Processing and Presentation as a Mechanism of Acquired Resistance to Immune Checkpoint Inhibitors in Lung Cancer. Cancer discovery. Available at:

765 http://cancerdiscovery.aacrjournals.org/lookup/doi/10.1158/2159-8290.CD-17-0593 [Accessed November 16, 2017].

Gong, C. et al., 2017. A computational multiscale agent-based model for simulating spatiotemporal tumour immune response to PD1 and PDL1 inhibition. Journal of the Royal Society, Interface, 14(134), p.20170320. Available at:

770 http://www.ncbi.nlm.nih.gov/pubmed/28931635 [Accessed November 27, 2017].

Gooden, M.J.M. et al., 2011. The prognostic influence of tumour-infiltrating lymphocytes in cancer: a systematic review with meta-analysis. British Journal of Cancer, 105(1), pp.93103. Available at: http://www.ncbi.nlm.nih.gov/pubmed/21629244 [Accessed September 21, 2017].

775 Goodman, A.M. et al., 2017. Tumor Mutational Burden as an Independent Predictor of Response to Immunotherapy in Diverse Cancers. Molecular Cancer Therapeutics, p.molcanther.0386.2017. Available at: http://www.ncbi.nlm.nih.gov/pubmed/28835386 [Accessed October 2, 2017].

Hanahan, D. \& Weinberg, R.A., 2011. Hallmarks of cancer: the next generation. Cell, 144(5), pp.646-674. Available at: http://www.ncbi.nlm.nih.gov/pubmed/21376230.

Hanahan, D. \& Weinberg, R.A., 2000. The hallmarks of cancer. Cell, 100(1), pp.57-70. Available at: http://www.ncbi.nlm.nih.gov/pubmed/10647931.

Hellerstein, M. et al., 1999. Directly measured kinetics of circulating T lymphocytes in normal and HIV-1-infected humans. Nature Medicine, 5(1), pp.83-89. Available at:

785 http://www.ncbi.nlm.nih.gov/pubmed/9883844 [Accessed September 27, 2017].

Hodi, F.S. et al., 2010. Improved Survival with Ipilimumab in Patients with Metastatic Melanoma. New England Journal of Medicine, 363(8), pp.711-723. Available at: http://www.ncbi.nlm.nih.gov/pubmed/20525992 [Accessed November 20, 2017].

Hugo, W. et al., 2016. Genomic and Transcriptomic Features of Response to Anti-PD-1 Therapy in Metastatic Melanoma. Cell, 165(1), pp.35-44. Available at: http://www.ncbi.nlm.nih.gov/pubmed/26997480 [Accessed November 17, 2017].

Jain, R.K., 1990. Delivery of Novel Therapeutic Agents in Tumors: Physiological Barriers and Strategies. JNCI: Journal of the National Cancer Institute, 81(8), pp.570-576. Available at: https://academic.oup.com/jnci/article-lookup/doi/10.1093/jnci/81.8.570 [Accessed 795 September 27, 2017].

Kelderman, S., Schumacher, T.N.M. \& Haanen, J.B.A.G., 2014. Acquired and intrinsic resistance in cancer immunotherapy. Molecular Oncology, 8(6), pp.1132-1139. Available at: http://www.ncbi.nlm.nih.gov/pubmed/25106088 [Accessed December 14, 2017].

Kirschner, D. \& Panetta, J.C., 1998. Modeling immunotherapy of the tumor - immune interaction. J. Math. Biol. Available at: https://link.springer.com/content/pdf/10.1007\%2Fs002850050127.pdf [Accessed April 9, 2018].

Kleffel, S. et al., 2015. Melanoma Cell-Intrinsic PD-1 Receptor Functions Promote Tumor 
Growth. Cell, 162(6), pp.1242-1256. Available at: http://www.ncbi.nlm.nih.gov/pubmed/26359984 [Accessed July 6, 2017].

Kodumudi, K.N. et al., 2016. Immune Checkpoint Blockade to Improve Tumor Infiltrating Lymphocytes for Adoptive Cell Therapy D. Chan, ed. PLOS ONE, 11(4), p.e0153053. Available at: http://www.ncbi.nlm.nih.gov/pubmed/27050669 [Accessed September 15, 2017].

810 Kosinsky, Y. et al., 2018. Radiation and PD-(L)1 treatment combinations: immune response and dose optimization via a predictive systems model. Journal for ImmunoTherapy of Cancer, 6(1), p.17. Available at: https://jitc.biomedcentral.com/articles/10.1186/s40425-018-0327-9 [Accessed April 9, 2018].

Kuznetsov, V.A. \& Knott, G.D., 2001. Modeling tumor regrowth and immunotherapy. Mathematical and Computer Modelling, 33(12-13), pp.1275-1287. Available at: http://www.sciencedirect.com/science/article/pii/S0895717700003149 [Accessed March 1, 2016].

Lai, X. \& Friedman, A., 2017a. Combination therapy for melanoma with BRAF/MEK inhibitor and immune checkpoint inhibitor: a mathematical model. Lai and Friedman BMC Systems Biology, 11. Available at: https://bmcsystbiol.biomedcentral.com/track/pdf/10.1186/s12918-017-04469? site $=$ bmcsystbiol.biomedcentral.com [Accessed November 27, 2017].

Lai, X. \& Friedman, A., 2017b. Combination therapy of cancer with cancer vaccine and immune checkpoint inhibitors: A mathematical model N. K. Haass, ed. PLOS ONE, 12(5), p.e0178479. Available at: http://dx.plos.org/10.1371/journal.pone.0178479 [Accessed November 27, 2017].

Le, D.T. et al., 2017. Mismatch repair deficiency predicts response of solid tumors to PD-1 blockade. Science (New York, N.Y.), 357(6349), pp.409-413. Available at: http://www.sciencemag.org/lookup/doi/10.1126/science.aan6733 [Accessed November 20, 830 2017].

Lindau, D. et al., 2013. The immunosuppressive tumour network: myeloid-derived suppressor cells, regulatory T cells and natural killer T cells. Immunology, 138(2), pp.105-15. Available at: http://www.ncbi.nlm.nih.gov/pubmed/23216602 [Accessed October 19, 2018].

835 Lindstrom, M.J. \& Bates, D.M., 1990. Nonlinear Mixed Effects Models for Repeated Measures Data. Biometrics, 46(3), pp.673-687. Available at: http://www.jstor.org/stable/2532087.

Malumbres, M. \& Barbacid, M., 2009. Cell cycle, CDKs and cancer: a changing paradigm. Nat Rev Cancer, 9(3), pp.153-166. Available at: http://www.ncbi.nlm.nih.gov/pubmed/19238148.

840 Mei, K.-C. et al., 2016. Investigating the effect of tumor vascularization on magnetic targeting in vivo using retrospective design of experiment. Biomaterials, 106, pp.276-85. Available at: http://www.ncbi.nlm.nih.gov/pubmed/27573135 [Accessed October 19, 2018].

Motzer, R.J. et al., 2015. Nivolumab versus Everolimus in Advanced Renal-Cell Carcinoma. New England Journal of Medicine, 373(19), pp.1803-1813. Available at: http://www.nejm.org/doi/10.1056/NEJMoa1510665 [Accessed November 27, 2017].

Nagy, J.A. et al., 2009. Why are tumour blood vessels abnormal and why is it important to know? British Journal of Cancer, 100(6), pp.865-869. Available at: http://www.ncbi.nlm.nih.gov/pubmed/19240721 [Accessed April 13, 2018]. 
Nani, F.K. \& Oğuztöreli, M.N., 1994. Modelling and simulation of Rosenberg-type adoptive cellular immunotherapy. IMA journal of mathematics applied in medicine and biology, 11(2), pp.107-47. Available at: http://www.ncbi.nlm.nih.gov/pubmed/8089590 [Accessed October 16, 2018].

Neumann, L. et al., 2010. Dynamics within the CD95 death-inducing signaling complex decide life and death of cells. Molecular systems biology, 6, p.352. Available at: http://www.ncbi.nlm.nih.gov/pubmed/20212524 [Accessed October 2, 2017].

Norris, S. et al., 2012. PD-1 Expression on Natural Killer Cells and CD8 ${ }^{+}$T Cells During Chronic HIV-1 Infection. Viral Immunology, 25(4), pp.329-332. Available at: http://www.ncbi.nlm.nih.gov/pubmed/22742708 [Accessed September 21, 2017].

860

Ott, P.A. et al., 2017. An immunogenic personal neoantigen vaccine for patients with melanoma. Nature, 547(7662), pp.217-221. Available at: http://www.nature.com/doifinder/10.1038/nature22991 [Accessed November 20, 2017].

Pardoll, D.M., 2012. The blockade of immune checkpoints in cancer immunotherapy. Nature Reviews Cancer, 12(4), pp.252-264. Available at: http://www.ncbi.nlm.nih.gov/pubmed/22437870 [Accessed September 21, 2017].

865 Parsa, A.T. et al., 2007. Loss of tumor suppressor PTEN function increases B7-H1 expression and immunoresistance in glioma. Nature medicine, 13(1), pp.84-88.

Peters, P.J. et al., 1991. Cytotoxic T lymphocyte granules are secretory lysosomes, containing both perforin and granzymes. The Journal of experimental medicine, 173(5), pp.1099-109. Available at: http://www.ncbi.nlm.nih.gov/pubmed/2022921 [Accessed September 25, 2017].

Pulluri, B. et al., 2017. Tumor microenvironment changes leading to resistance of immune checkpoint inhibitors in metastatic melanoma and strategies to overcome resistance. Pharmacological Research, 123, pp.95-102. Available at: http://www.ncbi.nlm.nih.gov/pubmed/28690075 [Accessed December 14, 2017].

875 Reck, M. et al., 2016. Pembrolizumab versus Chemotherapy for PD-L1-Positive Non-SmallCell Lung Cancer. New England Journal of Medicine, 375(19), pp.1823-1833. Available at: http://www.nejm.org/doi/10.1056/NEJMoa1606774 [Accessed November 14, 2016].

Rems, L. et al., 2013. Cell electrofusion using nanosecond electric pulses. Scientific Reports, 3(1), p.3382. Available at: http://www.ncbi.nlm.nih.gov/pubmed/24287643 [Accessed

$880 \quad$ September 15, 2017].

Ribas, A. et al., 2015. Pembrolizumab versus investigator-choice chemotherapy for ipilimumabrefractory melanoma (KEYNOTE-002): a randomised, controlled, phase 2 trial. The Lancet Oncology, 16(8), pp.908-918. Available at:

http://www.ncbi.nlm.nih.gov/pubmed/26115796 [Accessed November 20, 2017].

885 Rizvi, N.A. et al., 2015. Mutational landscape determines sensitivity to PD-1 blockade in nonsmall cell lung cancer. Science, 348(6230).

Rose, C. et al., 2018. Antigen-Specific Effector Cells That Traffic Tumors Increases the Generation of Tumor Local Radiation Therapy of B16 Melanoma. Available at: http://www.jimmunol.org/content/174/12/7516 [Accessed October 24, 2018].

890 Sade-Feldman, M. et al., 2017. Resistance to checkpoint blockade therapy through inactivation of antigen presentation. Nature communications, 8(1), p.1136. Available at: http://www.nature.com/articles/s41467-017-01062-w [Accessed December 5, 2017]. 
Salgado, R. et al., 2015. The evaluation of tumor-infiltrating lymphocytes (TILs) in breast cancer: recommendations by an International TILs Working Group 2014. Annals of Oncology, 26(2), pp.259-271. Available at: http://academic.oup.com/annonc/article/26/2/259/2800585/The-evaluation-oftumorinfiltrating-lymphocytes [Accessed September 21, 2017].

Sanchez-Paulete, A.R. et al., 2016. Cancer Immunotherapy with Immunomodulatory AntiCD137 and Anti-PD-1 Monoclonal Antibodies Requires BATF3-Dependent Dendritic Cells. Cancer Discovery, 6(1), pp.71-79. Available at: http://www.ncbi.nlm.nih.gov/pubmed/26493961 [Accessed September 28, 2017].

Scharping, N.E. et al., 2017. Efficacy of PD-1 Blockade Is Potentiated by Metformin-Induced Reduction of Tumor Hypoxia. Cancer immunology research, 5(1), pp.9-16. Available at: http://www.ncbi.nlm.nih.gov/pubmed/27941003 [Accessed April 5, 2018].

905 Serre, R. et al., 2016. Mathematical Modeling of Cancer Immunotherapy and Its Synergy with Radiotherapy. Cancer research, 76(17), pp.4931-40. Available at: http://www.ncbi.nlm.nih.gov/pubmed/27302167 [Accessed November 27, 2017].

Seymour, L. et al., 2017. iRECIST: guidelines for response criteria for use in trials testing immunotherapeutics. The Lancet. Oncology, 18(3), pp.e143-e152. Available at:

910 http://linkinghub.elsevier.com/retrieve/pii/S1470204517300748 [Accessed November 28, 2017].

Sharma, P. \& Allison, J.P., 2015. The future of immune checkpoint therapy. Science (New York, N.Y.), 348(6230), pp.56-61. Available at: http://www.ncbi.nlm.nih.gov/pubmed/25838373 [Accessed November 27, 2017].

915 Simpson, J.A.D. et al., 2010. Intratumoral T cell infiltration, MHC class I and STAT1 as biomarkers of good prognosis in colorectal cancer. Gut, 59(7), pp.926-33. Available at: http://www.ncbi.nlm.nih.gov/pubmed/20581241 [Accessed September 21, 2017].

Smahel, M., 2017. PD-1/PD-L1 Blockade Therapy for Tumors with Downregulated MHC Class I Expression. International journal of molecular sciences, 18(6).

920 Sunshine, J.C. et al., 2017. PD-L1 Expression in Melanoma: A Quantitative Immunohistochemical Antibody Comparison. Clinical Cancer Research, 23(16), pp.49384944. Available at: http://www.ncbi.nlm.nih.gov/pubmed/28428193 [Accessed November 21, 2017].

Taube, J.M., 2014. Unleashing the immune system: PD-1 and PD-Ls in the pre-treatment tumor microenvironment and correlation with response to PD-1/PD-L1 blockade.

Oncoimmunology, 3(11), p.e963413. Available at:

http://www.tandfonline.com/doi/abs/10.4161/21624011.2014.963413 [Accessed November 21, 2017].

Teng, M.W.L. et al., 2015. Classifying Cancers Based on T-cell Infiltration and PD-L1. Cancer research, 75(11), pp.2139-2145.

Topalian, S.L. et al., 2012. Safety, Activity, and Immune Correlates of Anti-PD-1 Antibody in Cancer. New England Journal of Medicine, 366(26), pp.2443-2454. Available at: http://www.nejm.org/doi/10.1056/NEJMoa1200690 [Accessed September 21, 2017].

Tsiatas, M., Mountzios, G. \& Curigliano, G., 2016. Future perspectives in cancer immunotherapy. Ann Transl Med, 4(14). Available at: http://dx.doi.org/10.21037/atm.2016.07.14 [Accessed November 22, 2017]. 
Yamamura, Y. et al., 2015. The key role of calreticulin in immunomodulation induced by chemotherapeutic agents. International Journal of Clinical Oncology, 20(2), pp.386-394. Available at: http://link.springer.com/10.1007/s10147-014-0719-x [Accessed September 15, 2017].

Zaretsky, J.M. et al., 2016. Mutations Associated with Acquired Resistance to PD-1 Blockade in Melanoma. New England Journal of Medicine, 375(9), pp.819-829. Available at: http://www.ncbi.nlm.nih.gov/pubmed/27433843 [Accessed November 20, 2017].

Zhou, F., 2009. Molecular mechanisms of IFN-gamma to up-regulate MHC class I antigen processing and presentation. International reviews of immunology, 28(3-4), pp.239-260. 